\title{
Revista de Medicina Veterinaria
}

January 2018

\section{Myxospóridos y nematodos en Hemidopsis microlepsis (Teleostei: Hemiodontidae) del río Gurguéia, Bom Jesús-Pi, Brasil}

\author{
Manoel Lopes da Silva Filho \\ Universidade Federal do Piauí, manoellopes@ufpi.edu.br \\ Glauciany Soares Lopes \\ Secretária da Educação do Estado do Piauí, glaucianylopes@hotmail.com \\ Daniela Kunkel \\ Centro de Ciências Agrárias/ Universidade Federal do Piauí, danikunkel@hotmail.com \\ Danilo Rodrigues Barros Brito \\ Instituto Federal de Educação Ciência e Tecnologia do Maranhão, danilobrito@ifma.edu.br
}

Follow this and additional works at: https://ciencia.lasalle.edu.co/mv

\section{Citación recomendada}

Lopes da Silva Filho M, Lopes GS, Kunkel D y Barros Brito DR. Myxospóridos y nematodos en Hemidopsis microlepsis (Teleostei: Hemiodontidae) del río Gurguéia, Bom Jesús-Pi, Brasil. Rev Med Vet. 2018;(36):

89-96. doi: https://doi.org/10.19052/mv.5175 


\title{
Mixosporídeos e nematóides em Hemiodopsis microlepis (Teleostei: Hemiodontidae) do rio Gurguéia-Bom Jesus-Pi, Brasil
}

\author{
Manoel Lopes da Silva Filho1 / Glauciany Soares Lopes² / Daniela Kunkel ${ }^{3}$ / \\ Danilo Rodrigues Barros Brito ${ }^{4}$
}

1 Médico Veterinário, Mestre em Ciência Animal, Doutor em Ciências Veterinárias, Professor Adjunto / Depto. de Reprodução Animal / Campus Professora Cinobelina Elvas - Universidade Federal do Piauí.

$\triangle$ manoellopes@ufpi.edu.br

2 Biologa, Mestre em Fitotecnia, Professora da Secretária da Educação do Estado do Piauí - SEDUC. $\triangle$ glaucianylopes@hotmail.com

3 Médica Veterinária, Mestre em Zootecnia, Doutoranda em Ciência Animal- Centro de Ciências Agrárias/ Universidade Federal do Piauí, bolsista CNPq.

$\triangle$ danikunkel@hotmail.com

4 Médico Veterinário, Mestre, Doutor em Ciência Animal, Professor/ Departamento de Desenvolvimento Educacional/ Instituto Federal de Educação Ciência e Tecnologia do Maranhão.

$\bowtie$ danilobrito@ifma.edu.br

Como citar este artigo: Filho ML, Lopes GS, Kunkel D, Barros Brito M. Mixosporídeos e nematóides em Hemiodopsis microlepis (Teleostei: Hemiodontidae) do rio GurguéiaBom Jesus-Pi, Brasil. Rev Med Vet. 2018;(36):89-96. doi: http://dx.doi. org/10.19052/mv.5175

\section{Resumo}

O objetivo deste estudo foi realizar um estudo ictioparasitológico sobre a espécie $\mathrm{He}$ miodopsis microlepis (peixes de arqueiros), do rio Gurguéia, situada em Bom Jesus-Piauí, Brasil. Coletaram-se trinta espécimenes de Hemiodopsis microlepis, aos quais realizou-se uma análise morfológico externo e coleta de pequenos fragmentos das brânquias, fígado, rins e intestinos para identificar os parasitas Myxobolus sp., Henneguya sp. e Camallanus sp. em um microscópio óptico (40X). Aproximadamente $90 \%$ dos peixes examinados foram parasitados por myxosporidos pertencentes à família Myxobolidae, dos quais ao redor do $20 \%$ foram parasitados por Henneguya sp. e o $70 \%$ foram parasitados por Myxobolus sp. Solo o 5\% das espécimenes parasitados por Myxobolus sp. foram também parasitados por nematódeos. O estudo de ictioparasitologia realizado sobre a espécie Hemiodopsis microlepis confirmou a presença de parasitas Myxobolus sp. e Henneguya e os nematódeos da espécie Camallanus sp.

Palavras chave: apicomplexa, archer peixes, ictioparasitologia, Myxozoa, nematódeos.

\section{Myxosporids and nematodes in Hemiodopsis microlepis (Teleostei: Hemiodontidae) of the Gurguéia River, Bom Jesus-Piauí, Brazil}

\begin{abstract}
The objective of this study was to carry out an ichthyoparasitological study on the species Hemiodopsis microlepis (archerfish) of the Gurguéia River, located in Bom JesusPiauí, Brazil. Thirty specimens of Hemiodopsis microlepis were collected, which underwent an external morphological analysis and collection of small fragments of gills, liver, kidneys, and intestines to identify the parasites Myxobolus sp., Henneguya sp., and Camallanus sp., using an optical microscope (40X). About $90 \%$ of the examined fish were parasitized by myxosporids belonging to the Myxobolidae family, of which around $20 \%$ were parasitized by Henneguya sp., and 70\% were parasitized by Myxobolus sp. Only 5\% of the specimens parasitized by Myxobolus sp. were also parasitized by nematodes. The ichthyoparasitological study of the species Hemiodopsis microlepis confirmed the presence of the parasites Myxobolus sp. and Henneguya sp., as well as the nematodes of the species Camallanus sp.
\end{abstract}

Keywords: Apicomplexa, archerfish, ichthyoparasitism, Myxozoa, nematodes. 


\title{
Myxospóridos y nematodos en Hemidopsis microlepsis (Teleostei: Hemiodontidae) del río Gurguéia, Bom Jesús-Pi, Brasil
}

\begin{abstract}
Resumen
El objetivo de este estudio fue realizar un estudio ictioparasitológico sobre la especie Hemiodopsis microlepis (peces de arqueros), del río Gurguéia, situada en Bom JesusPiauí, Brasil. Se recolectaron treinta especímenes de Hemiodopsis microlepis, a los que se les realizó un análisis morfológico externo y recolección de pequeños fragmentos de las branquias, hígado, riñones e intestinos para identificar los parásitos Myxobolus sp., Henneguya sp. y Camallanus sp. en un microscopio óptico (40X). Alrededor del $90 \%$ de los peces examinados fueron parasitados por myxosporidos pertenecientes a la familia Myxobolidae, de los cuales alrededor del $20 \%$ fueron parasitados por Henneguya sp. y el $70 \%$ fueron parasitados por Myxobolus sp. Solo el 5\% de los especímenes parasitados por Myxobolus sp. fueron también parasitados por nematodos. El estudio ictioparasitológico realizado sobre la especie Hemiodopsis microlepis confirmó la presencia de parásitos Myxobolus sp. y Henneguya y los nematodos de la especie Camallanus sp.
\end{abstract}

Palabras clave: apicomplexa, archer peces, ictioparasitismo, Myxozoa, nematodos.

\section{INTRODUÇÃO}

Os myxosporeos são parasitos encontrados comumente em peixes formando cistos nas brânquias, órgãos internos e na musculatura. Estes cistos contêm numerosos esporos. Espécies de três gêneros são as mais comuns em peixes marinhos e de água doce: Myxobolus Bütschli, 1882, Henneguya Thélohan, 1892 e Kudoa (1). A maioria destes parasitos (aproximadamente 1400 espécies conhecidas) é estenoxeno, atacando somente uma única espécie. Entretanto, uma espécie de peixe pode albergar dezenas de espécies de mixosporeo (2). Uma parte das espécies é conhecida como parasitas patogênico. Alguns deles provocam doenças específicas nos alevinos das espécies regionais e exóticas (2).

Desde o fim do século dezenove, vários grupos de especialistas estão pesquisando o ciclo evolutivo dos mixosporeos e as doenças que eles provocam. Apesar disso, há apenas quinze anos que obtiveram resultados significativos possibilitando explicar os processos patogênicos no hospedeiro. Pelo conhecimento atual, os mixosporeos são organismos metazoários primitivos concluindo uma fase vegetativa prolongada nos peixes onde surgem esporos apresentando no mínimo seis células. As formas vegetativas geralmente são plasmódios de tamanho grande contendo núcleos vegetativos e células germinativas (2).

Os conhecimentos sobre a morfologia, taxonomia e o ciclo vital dos mixosporideos dentro do organismo dos peixes, enriqueceram bastante nas últimas décadas (3). O ciclo envolve dois hospedeiros: um vertebrado (peixe) e um invertebrado (o anelídeo Tubifex tubifex) (4). Em cada um desses hospedeiros encontram-se esporos com características diferentes. O local de desenvolvimento dos esporos é a cartilagem do hospederio, com preferência pelos alevinos, existindo destruição do tecido (5). Quando se localizam na zona posterior da vértebra, exercem pressão sobre o nervo que controlam as células pigmentares da zona da cauda, ficando esta intensamente enegrecida. Se localizados perto da cápsula auditiva provocam distúrbios natatórios característicos (6).

Numerosas descrições de myxosporeos foram relatadas em peixes de diferentes áreas geográficas $(7,8)$. O gêne- 
ro mais rico dos myxosporeos é o Myxobolus apresentando mais de 450 espécies. Os resultados recentes das pesquisas na área foram relatados por Lom et al, (1992) (8). Até o presente momento, dezenove espécies de peixes brasileiros têm sido descritas como hospedeiro de diferentes espécies Myxobolus (9-17). No entanto, há poucos estudos sobre ultraestrutura de representantes de Myxobolus sp. do Brasil, do qual apenas três espécies têm sido descritas (18).

Representantes do gênero Henneguya são os mais estudados. Apresentam esporo alongado e dois filamentos polares longos. Formam seus cistos preferencialmente nos filamentos e arcos branquiais, provocando maior contato entre as lamelas secundárias diminuindo desta forma a superfície de absorção na extremidade dos filamentos, podendo chegar a provocar hiperplasia e hipertrofia dos órgãos infectados $(19,20,21)$.

Muitos representantes do filo Nematoda são também relatados em várias espécies, sendo inclusive o maior dos grupos de parasitos de peixes. Mas, apesar disso são considerados, de maneira geral, espécies pouco patogênicas. São fáceis de serem reconhecidos devido ao formato alongado com extremidades afiladas. São dióicos e exibem dimorfismo sexual. Apresentam ciclo indireto, com participação de copépodes planctônicos como hospedeiros intermediários. Especial menção é feita para os camallanídeos de peixes de água doce, nematódeo que habita o reto (porção terminal do intestino grosso) dos peixes. O espécime parasitado apresenta protusão dos vermes pelo ânus, quando os peixes se movimentam os vermes retornam ao reto. Em algumas espécies não observamos esse sinal. Ainda podemos observar emagrecimento, deformações da coluna e nado lento $(22,23,24)$. No Brasil existem relatos sobre a presença de espécies de Philometra em peixes de água doce e marinhos, estas são normalmente encontradas encistadas na pele, nadadeiras, ovário e cavidade corporal provocando peritonite $(19,25)$.

A espécie Hemiodopsis microlepsis, conhecido popularmente como peixe flecheiro ou voador, de maneira geral, possui importância econômica insignificante, entretanto para a população ribeirinha e/ou carente representa uma importante fonte de alimentação. $\mathrm{O} \mathrm{He}$ miodopsis microlepsis apresenta corpo roliço e baixo com porte médio de até $25 \mathrm{~cm}$ e altura contida, cerca de 4 vezes no comprimento padrão, linha lateral com cerca de 120 escamas, 26 séries de escamas entre a dorsal e a linha lateral e 16 entre esta e a base da ventral, uma mancha escura alongada no flanco, na região posterior da nadadeira dorsal, com diâmetro aproximadamente do mesmo tamanho do olho e a qual é eventualmente seguida por uma faixa escura inconspícua, que vai até o final do pedúnculo caudal e lóbulo inferior da nadadeira caudal com uma faixa amarelada (26).

O objetivo do presente trabalho é fazer um estudo ictioparasitológico na espécie Hemiodopsis microlepsis (peixe flecheiro), que por sua vez é utilizado como fonte de alimentação pela população ribeirinha do Rio Gurguéia, localizado na cidade de Bom Jesus-Piauí.

\section{MATERIAL E MÉTODOS}

Foram coletados trinta espécimes de Hemiodopsis microlepis Kner, 1859 (Teleostei: Hemiodontidae) conhecido pelos nomes vulgares de "flecheiro" e "voador", no rio Gurguéia, na cidade de Bom Jesus-Piauí localizada na Região Sudoeste do estado do Piauí, NE, a $635 \mathrm{~km}$ da capital Teresina, engloba uma área de $5.469,156 \mathrm{~km}^{2}$. Sua posição geográfica é definida pelas coordenadas: latitude $09^{\circ} 04^{\prime} 28^{\prime \prime}$ sul e a uma longitude $44^{\circ} 21^{\prime} 31^{\prime \prime}$ oeste, estando a uma altitude de $277 \mathrm{~m}$ (27).

Os espécimes capturados foram transportados vivos ao Laboratório de Sanidade Animal do Campus Professora Cinobelina Elvas da Universidade Federal do Piauí (CPCE/UFPI), sendo mantidos em tanques.

Os peixes foram eutanasiados por um médico veterinário, pela imersão dos animais em solução de hidrocloridrato de benzocaína e água, tamponada com $\mathrm{pH}$ neutro, na dose recomendada de $250 \mathrm{mg} / \mathrm{L}$. A utilização de hidrocloridrato de benzocaína proporciona a morte do animal mais rapidamente, com uma ótima eficiência e 
estética. Imediatamente após a observação da ausência dos reflexos oculopalpebrais e conclusão do processo de eutanásia, foram observadas em lupa as nadadeiras e em seguida feito um corte, retirando-se o opérculo para exposição das brânquias.

Após a observação das brânquias fez-se uma incisão na cavidade celomática até o ânus, ao longo da linha média ventral, expondo as vísceras, rim e fígado. Foram retirados pequenos fragmentos das brânquias, fígado, rins e intestinos, colocados em lâminas e cobertos por lamínulas e examinados ao microscópio de luz com objetiva de $40 X(17)$.

Os parasitos encontrados pertencentes à classe Myxosporeos foram fixados com Glutaraldeído a $24,5 \%$, em $24 \mathrm{~h}$, sendo o mesmo utilizado como $1^{\circ}$ fixador em concentrações variáveis de $2 \%$ a $5 \%$ e depois passado ao tampão de cacodilato de sódio a 0,2 M com pH 7,0-7,2 para estudo morfológico dos esporos e os pertencentes à classe Nematódeos fixados em AFA.

\section{Resultados}

Nos peixes examinados, foram registradas duas morfoespécies de parasitos pertencentes às classes Myxosporeos e Nematódeos. Dos espécimes de peixes examinados cerca de $85 \%$ se encontravam parasitados por pelo menos um dos tipos encontrados. Do total parasitado cerca de $90 \%$ apresentavam-se parasitados por mixosporídeos pertencentes à família Myxobolidae. Dos exemplares parasitados por Myxosporideos cerca de $20 \%$ estavam parasitados por Henneguya sp. em seus filamentos branquiais e no fígado, $70 \%$ encontravam-se parasitados por Myxobolus sp. em forma de cisto no fígado e isolados nas brânquias, fígado e vesícula biliar. Apenas 5\% dos espécimes, que também estava parasitado por Myxobolus sp., encontravam-se parasitado por Nematódeo, localizado no intestino (tabela 1), que de acordo com suas características foi identificado como Camallanus sp. (figura 1).

Tabela 1. Percentual de parasitos encontrados de H. microlepis, prevalência de cada parasito e órgãos parasitados.

Bom Jesus-PI, 2015

\begin{tabular}{|c|c|c|c|c|}
\hline Hospedeiro & Total de amostras parasitadas & Parasitos & Prevalência & Órgão parasitado \\
\hline \multirow{7}{*}{ H. microlepis } & \multirow{7}{*}{$85 \%$} & \multirow{4}{*}{ Mixobolus sp. } & \multirow{4}{*}{$70 \%$} & Fígado $(C)$ \\
\hline & & & & Fígado (I) \\
\hline & & & & Brânquias (I) \\
\hline & & & & V. biliar (I) \\
\hline & & \multirow{2}{*}{ Henneguya sp. } & \multirow{2}{*}{$25 \%$} & Fígado (I) \\
\hline & & & & Brânquias (I) \\
\hline & & Camallanus sp. & $5 \%$ & Intestinos \\
\hline
\end{tabular}

OP: órgãos parasitados; TAP: total de amostras parasitadas; Prev: prevalência; C: cisto; I: isolados. 
Figura 1. Camallanus sp. encontrado no intestino de H. microlepis no rio Gurguéia

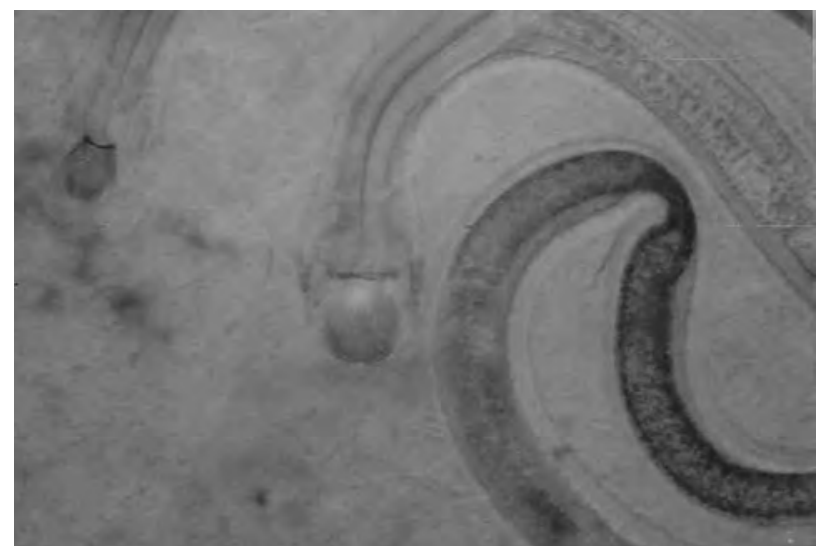

\section{Discussão}

Os espécimes de mixosporidios são encontrados na maioria dos órgãos do corpo de seus hospedeiros, sendo mais comuns na bexiga natatória, vesícula biliar, cérebro e brânquias (28), apresentado um alto grau de tropismo, tanto para o hospedeiro, como para o tecido infectado (29).

Os resultados obtidos demonstraram grande semelhança com os obtidos por Olmos et al. (30) em exames realizados com espécimes de Trichomycterus aerolatus Valenciennes, 1848 (Siluriformes), Diplomystes nahuelbutaensis Arratia, 1987 (Siluriformes) e Percilia irwini Eigenmann, 1927 (Perciformes), nativos da bacia do rio Laja no Chile, cujos exemplares apresentavam cérebro, coração, fígado, brânquias e intestinos parasitados por Myxobolus sp., Henneguya sp. e por nematódeos. No hemisfério norte, Henneguya sp. é considerado um patógeno severo, e significa um risco no cultivo de peixes siluriformes (ordem que inclui as espécies chilenas $T$. areolatus, D. nahuelbutaensis). Com respeito aos nematóides, estes são encontrados com freqüência no intestino e eles são considerados patógenos fracos. Alves et al. (31) examinaram 30 peixes da espécie Poecilia reticulata Peters, 1859 provenientes de psicultura ornamental, verificando que $93,4 \%$ dos peixes estavam parasitados por Camallanus contti Fujita, 1927. Entretanto, no presente estudo foram encontrados somente $5 \%$ das amostras parasitadas por Camallanus sp., que encontrou a presença de 5,9\% do parasito Camallanus maculatus n. sp. Martins, 2007 nas espécies de peixes Xiphophorus maculatus Günther, 1866, P. reticulata e Poecilia Sphenops Valenciennes, 1846 provenientes de água doce no estado de Santa Catarina.

Em uma análise histológica realizada nas brânquias de Prochilodus lineatus Valenciennes, 1847 provenientes do rio do Peixe, São Paulo, revelou numerosos cistos de Myxobolus sp. bem delimitados na base dos filamentos branquiais, na região da musculatura estriada próximo às artérias brânquias, porém nenhuma resposta inflamatória pronunciada foi encontrada no local de infecção (32). Em outros estudos realizados encontraram parasitos Myxobolus parasitando o coração de Pimelodus ornatus Kner, 1858 (33), na "patinga" hibrida (Piaractus Mesopotamicus Holmberg, 1887 x Piaractus Brachypomus Cuvier, 1818) foram encontrados parasitas no rim e no baço (34), entretanto no presente estudo realizado na espécie H. microlepis havia a presença do Myxobolus sp. parasitando de forma isolada as brânquias, vesícula biliar e o fígado, o qual também apresentava cistos com o mesmo parasito.

Em relação ao Henneguya sp. foram isolados nas brânquias e no fígado da espécie $H$. microlepis, corroborando com Azevedo et al. (35) em pesquisas realizadas com Hemiodopsis microlepsis também no rio Poty, encontraram uma nova espécie de Henneguya, Henneguya hemiodopsis, a qual infecta as lamelas branquiais ocasionando atrofia local e deformação das mesmas e com por Mendonça et al. (36) em pesquisas realizadas com Pimelodus argenteus Perugia, 1891 provenientes do rio Poty, Teresina, PI, cujos resultados demonstram que a espécie apresentava-se parasitada por Henneguya sp. em seus filamentos branquiais.

Algumas espécies de Henneguya levam a patogenia em seus hospedeiros. No entanto, na maioria das espécies ocorre pouca ou nenhuma resposta do hospedeiro e a condição mais encontrada nas análises histológicas é o encapsulamento do plasmódio por tecido conjuntivo e 
fibrótico isolando o parasito e prevenindo sua dispersão para os tecidos adjacentes (37).

Entretanto, a importância da patogenicidade não esta apenas no caráter letal da doença, mas também no grau da lesão causada a alguns tecidos. Esse fato deve ser levado em consideração porque os hospedeiros dos mixosporidios são os peixes e, os mesmos constituem um valioso artigo comercial para o homem (38).

\section{Conclusão}

O estudo ictioparasitológico realizado na espécie $\mathrm{He}$ miodopsis microlepis (peixe flecheiro, peixe-voador), comprovou a presença dos parasitos Myxobolus sp., Henneguya sp. e nematoides como o Camallanus sp.

\section{Agradecimento}

A Carlos Azevedo da Universidade de Porto, Portugal, pela grande ajuda.

\section{REFERÊNCIAS}

1. Luque JL. Biologia, epidemiologia e controle de parasitos de peixes. Rev Bras Parasitol Vet. 2004;13(1):161-5.

2. Lászlo B, Csaba S, Kálmán M. Atuais conhecimentos sobre Myxosporea (Myxozoa), parasitas de peixes: Um estágio alternativo dos parasitas no Brasil. Braz J Vet Res Anim Sci. 2002;39(5):271-6. https://doi. org/10.1590/S1413-95962002000500010

3. Pavanelli GC, Eiras JC, Takemoto RM. Doenças de peixes. Maringá: Nupelia; 1998.

4. Kent M L, Andree KB, Bartholomew JL, Elmatbouli M, Desser SS, Devlin RH, et al. Recent advances in our knowledge of the Myxozoa. J Eukaryot Microbiol. 2002;48(4):395-413. https://doi.org/10.1111/j.1550-7408.2001.tb00173.x

5. Thompson KG, Nehring RB, Bowden DC, Wygant T. Response of rainbow trout Oncorhynchus mykiss to exposure to Myxobolus cerebralis above and below a point source of infectivity in the upper Colorado River. Dis Aquat Organ. 2002;49(3):171-8.

6. Pavanelli GC, Eiras JC, Takemoto RM. Doenças de peixes. Profilaxia, diagnóstico e tratamento. Editora Universidade de Estadual de Maringá; 2002.

7. Landsberg JH, Lom J. Taxonomy of the genera Myxobolus/Myxosoma group (Myxobolidae: Myxosporea), current listing of species and revision of synonyms. Syst Parasitol. 1991;18(3):165-86. https:// doi.org/10.1007/BF00009358

8. Lom J, Dykova, I. Protozoan parasites of fishes. Elsevier Science Publisher; 1992.

9. Walliker D. Myxosporidea of some Brazilian freshwater fishes. J Parasitol. 1969;55(5):942-8. https://doi. org/10.2307/3277155

10. Kent ML, Hoffman GL. Two new species of Myxozoa, Myxobolus inaequus sp. n. and Henneguya theca sp. n. from the brain of a South America knife fish, Eigenmannia virescens (V.). J Protozool. 1984;31(1):91-4. https://doi.org/10.1111/j.1550-7408.1984.tb04295.x

11. Molnár K, Békési L. Description of a new Myxobolus species, M. colossomatis n. sp. from the teleost Colossoma macropomum of the Amazon River basin. J Appl Ichthyol. 1993;9(1):57-63. https://doi. org/10.1111/j.1439-0426.1993.tb00388.x

12. Gioia I, Cordeiro NS. Brazilian myxosporidians' checklist (Myxozoa). Acta Protozool. 1996;35:137-149.

13. Casal G, Matos E, Azevedo C. Ultrastructural data on the life stages of Myxobolus braziliensis n. sp., parasite of an Amazonian fish. Eur J Protistol. 1996;32(1):1237. https://doi.org/10.1016/S0932-4739(96)80047-2

14. Molnár K, Ranzani-Paiva MJ, Eiras JC, Rodrigues EL. Myxobolus macroplasmodialis sp. n. (Myxozoa: Myxosporea), a parasite of the abdominal cavity of the characid teleost, Salminus maxillosus, in Brazil. Acta Protozool. 1998;37(4):241-5.

15. Adriano EA, Arana S, Ceccarelli PS, Cordeiro NS. Light and scanning microscopy of Myxobolus porofilus sp. n. (Myxosporea: Myxobolidae) infecting the visceral cavity of Prochilodus lineatus (Pisces: Characiformes: Prochilodontidae) cultivated in Brazil. Folia Parasitol (Praha). 2002;49(4):259-62. https://doi. org/10.14411/fp.2002.049 
16. Azevedo C, Corral L, Matos E. Myxobolus desaequalis n. sp. (Myxozoa, Myxosporea), parasite of the Amazonian freshwater fish, Apteronotus albifrons (Teleostei, Apteronotidae). J Eukaryot Microbiol. 2002;49(6):485-8. https://doi. org/10.1111/j.1550-7408.2002.tb00233.x

17. Cellere EF, Cordeiro NS, Adriano EA. Myxobolus absonus sp.n. (Myxozoa: Myxosporea) parasitizing Pimelodus maculatus (Siluriformes: Pimelodidae), a South American freshwater fish. Mem Inst Oswaldo Cruz. 2002;97(1):79-80. https://doi.org/10.1590/ S0074-02762002000100012

18. Casal G, Matos E, Azevedo C. Ultrastructural data on the spore of Myxobolus maculatus n. sp. (phylum Myxozoa), parasite from the Amazonian fish Metynnis maculatus (Teleostei). Dis Aquat Organ. 2002;51(2):107-12. https://doi.org/10.3354/ dao051107

19. Eiras, JC, Pavanelli, GC, Ranzani-Paiva, MJT, Takemoto, RM. Gill histopathology of piaractus mesopotamicus (Osteichthyes:Serraslmidae) infected by Henneguya piaractus Martins \& Sousa, 1997 (Myxobolidae). Res Review Parasitol. 1999;59(3-4):117-20.

20. Eiras, JC. Synopsis of the species of the genus Heneneguya Thélohan, 1892 (Myxozoa: Myxosporea: Myxobolidae). Syst Parasitol. 2002;52(1):43-54. https://doi.org/10.1023/A:1015016312195

21. Ferraz E, Thatcher, VE. Camallanus acaudatus sp.n. (Nematoda: Camallanidae) é uma descrição do macho de Camallanus tridentatus (D.,1884) parasitas de peixes da Amazônia brasileira. Amazoniana. 1990;11(12):135-45.

22. Alves DR, Luque JL, Paraguassú AR. Ectoparasitos da tilápia nilótica Oreochromis niloticus (Osteichthyes: Cichlidae) da Estação de Piscicultura da UFRRJ. Rev Univ Rural-Sér Ciên da Vida. 2000;22(1):81-5.

23. Levsen A. Transmission ecology and larval behaviour of Amallanus cotti (Nematotoda, Camallanidae) under aquarium conditions. Aquarium Sci Conservat. 2001;3(4):315-25. https://doi. org/10.1023/A:1013137801600

24. Luque JL, Alves DR, Ribeiro RS. Community ecology of the metazoan parasites of banded croaker, Paral- onchurus brasiliensis (Osteichthyes: Sciaenidae) from the coastal zone of the State of Rio de Janeiro, Brasil. Acta Scientiarum. 2003;25(2):273-8.

25. Langeani Neto F. Estudo Filogenético e revisão taxonômica da Família Hemiodontidae Boulenger, 1904 (sensu Roberts, 1794) (Ostariophysi, Characiformes) [tese de doutorado]. Universidade de São Paulo; 1996.

26. Vazzoler AEAM. Biologia da reprodução de peixes teleósteos: teoria e prática. Maringá: Eduem-São Paulo: SBI; 1996.

27. Instituto Brasileiro de Geografia e Estatística. Banco de dados agregados [internet]. 2012 [citado 2015 ago 15]. Disponível em: http://www.sidra.ibge.gov.br/bda/pecua/default.asp?t=2\&z=t\&o=22\&u1=1\&u3=1\&u4=1\& $5=1 \& u 6=1 \& u 7=1 \& u 2=1$

28. Lom J. Notes on the ultraestructure and sporoblast development in fish parasitizing myxosporidian of the genus Sphareomyxa. Z Zellforsch Mikrosk Anat. 1969;97(3):416-37. https://doi.org/10.1007/ BF00968848

29. Salim KY, Desser SS. Description and phylogenetic systematic of Myxobolus ssp. from Cyprinids in Algoniun Park, Ontario. J Eukariot Microbiol. 2000;47(3):30918. https://doi.org/10.1111/j.1550-7408.2000. tb00052.x

30. Olmos VL, Victoriano P, Habit E, Valdovinos C. Parásitos de peces nativos de la ceunca Del Río Laja (Chile Central) y alcances sobre sus ciclos de vida. Ach Med Vet. 2003;35(2):195-203. https://doi.org/10.4067/ S0301-732X2003000200007

31. Alves DR, Luque JL, Paraguassú AR, Marques FA. Ocorrência de Camallanus conti (Nematoda: Camallanidae) parasitando o Guppy, Poecilia reticulata (Osteichthyes: Poecillidae) no Brasil. Rev Univ Rural-Sér Ciên da Vida. 2000;22(supl.):77-9.

32. Vieira DHHD. Análises morfológicas dos mixozoários (Myxozoa) parasitos presentes em tecido nervoso de peixes coletados no Rio do Peixe, médio Rio Tietê, São Paulo, Brasil [trabalho de conclusão de curso]. Botucatu: Universidade Estadual de São Paulo; 2013.

33. Matos E, Videira M, Velasco M, Sanches O, São Clemente SC, Matos P. Infection of the heart of Pimelodus ornatus (Teleostei, Pimelodidae), by Myxobolus 
sp (Myxozoa, Myxobolidae). Braz J Vet Parasitol. 2014;23(4):543-56. https://doi.org/10.1590/S198429612014083

34. Franceschini L, Zago AC, Schalch SHC, Garcia F, Romera DM, Silva RJ. Parasite infections of Piaractus mesopotamicus and hybrid (P. mesopotamicus $\mathrm{x}$ Piaractus brachypomus) cultured in Brazil. Rev Bras Parasitol Vet. 2013;22(3):407-14. https://doi. org/10.1590/S1984-29612013000300015

35. Azevedo C, Casal G, Mendonça I, Matos E. Fine structure of Henneguya hemiodopsis sp. n. (Myxozoa), a aprasite of the gills of the Brazilian teleostean fish Hemiodopsis microlepes (Hemiodoptidae). Mem Inst Oswaldo Cruz. 2009;104(7):975-9. https://doi. org/10.1590/S0074-02762009000700006
36. Mendonça IL, Muratori MCS, Araripe MNBA, Matos E. Aspectos morfológicos de Mixosporídeo Henneguya parasitando Pimelodus argentens. In: XXIV Congresso Brasileiro de Zoologia, 2002, Itajaí, SC. Anais do evento. Itajaí, SC: Sociedade Brasileira de Zoologia.

37. Barassa B, Adriano E, Cordeiro NS, Arana S, Ceccarelli OS. Morphology and host-parasite interaction of Henneguya azevedoi n. sp. parasite of gills of Leporinus obtusidens from Mogi Guaçu River, Brazil. Parasitol Res. 2012;110(2):887-94. https://doi.org/10.1007/ s00436-011-2571-5

38. Silva Junior ACS. Myxosporidiose em peixes de água doce. Estação Científica (Unifap). 2012;2(2):25-39. 